\title{
CERTIFICATS VERTS OU PRIME DE RACHAT ? ÉVOLUTION DU MARCHÉ DES CERTIFICATS VERTS EN WALLONIE
}

\author{
Nicolas Boccard et Axel Gautier
}

De Boeck Supérieur | «Reflets et perspectives de la vie économique »

2019/1 Tome LVII | pages 163 à 171

ISSN 0034-2971

ISBN 9782807393295

Article disponible en ligne à l'adresse :

https://www.cairn.info/revue-reflets-et-perspectives-de-la-vieeconomique-2019-1-page-163.htm

Distribution électronique Cairn.info pour De Boeck Supérieur.

(C) De Boeck Supérieur. Tous droits réservés pour tous pays.

La reproduction ou représentation de cet article, notamment par photocopie, n'est autorisée que dans les limites des conditions générales d'utilisation du site ou, le cas échéant, des conditions générales de la licence souscrite par votre établissement. Toute autre reproduction ou représentation, en tout ou partie, sous quelque forme et de quelque manière que ce soit, est interdite sauf accord préalable et écrit de l'éditeur, en dehors des cas prévus par la législation en vigueur en France. Il est précisé que son stockage dans une base de données est également interdit. 


\section{Certificats verts ou prime de rachat? Évolution du marché des certificats verts en Wallonie Green certificates or feed-in premiums? The changes in the green certificate market in Wallonia}

Nicolas Boccard ${ }^{1}$, Axel Gautier²

Résumé - La Wallonie a mis en place en 2003 un mécanisme de certificats verts échangeables pour promouvoir le développement des énergies issues de sources renouvelables. Le marché des CV s'est trouvé dès 2007 en déséquilibre avec une offre excédentaire de certificats du fait notamment du soutien différencié et généreux à la filière solaire photovoltaïque. Ce déséquilibre persistant a fait chuter le prix des CV à un prix proche du prix de rachat garanti de $65 €$. Dans les faits, le mécanisme des certificats verts s'est transformé en une prime de rachat garantie qu'il est aujourd'hui nécessaire de modifier.

Mots clés : énergie renouvelable, certificats verts, prime de rachat

Abstract - In 2003, Wallonia implemented a mechanism of exchangeable green certificates to promote the development of renewable energies. Since 2007, the green certificate market has been in disequilibrium with an excess supply of certificates mainly due to the differentiated and generous support given to the solar photovoltaic sector. This persistent disequilibrium has made the price of green certificates plummet to close to their guaranteed purchase price of $€ 65$. In reality, the green certificate mechanism has become a guaranteed feed-in premium that must now be modified.

Keywords: renewable energy, green certificates, feed-in premium

1. Nicolas Boccard est professeur à l'Université de Gérone (Espagne).

Email : nicolas.boccard@udg.edu.

2. Axel Gautier est professeur à HEC école de gestion de l'Université de Liège.

Email : agautier@uliege.be. 


\section{INTRODUCTION}

Les certificats verts ont pour objectif de soutenir la production d'énergie issue de sources renouvelables (solaire, éolien, biomasse, hydro, cogénération). Dans Gautier et Boccard (2015), nous avons calculé le coût pour la période 2003-2012 du mécanisme de certificats verts en place en Wallonie. Nous concluons que le subside octroyé aux producteurs par les certificats verts est considérable, notamment pour la filière photovoltaïque. En effet, le subside au photovoltaïque pour la période considérée était égal à $588 €$ par MWh produit, ce qui équivaut à un coût de $2332 €$ par tonne de $\mathrm{CO}_{2}$ évitée, au vu du prix moyen sur le marché durant la période. Les subsides octroyés aux autres filières de production renouvelables sont également importants mais plus proches, par comparaison, des soutiens accordés dans les pays voisins.

L'objectif du présent article est d'évaluer le fonctionnement du marché des certificats verts wallons à ce jour. Nous comparons, dans une première section, les différents mécanismes de soutien aux énergies renouvelables : les certificats verts échangeables (CV), le prix de rachat garanti (ou feed-in tariff, FiT) et la prime de rachat garantie (ou feed-in premium, FiP). Ces trois schémas de soutien se distinguent par leur couverture des risques liés à la technologie utilisée. Les producteurs d'énergie renouvelable font en effet face à deux risques : un risque de marché relatif à l'évolution du prix de l'énergie et un risque technologique lié à l'évolution des coûts des moyens de production et des performances des installations de production. Le FiT assure le producteur contre ces deux risques, le FiP contre le risque technologique le certificat vert n'assure contre aucun des deux risques.

Dans une deuxième section, nous analysons le mécanisme mis en place en Wallonie et le fonctionnement de ce marché. Celui-ci est caractérisé à partir de 2007 par un excès d'offre. Ce déséquilibre s'est accru jusqu'en 2012 avant de diminuer progressivement tout en restant important avec, en 2017, une quantité de CV octroyée une fois et demi supérieure à la demande et un stock considérable de CV accumulés. En parallèle, le prix a diminué et il est aujourd'hui proche du prix de rachat garanti. Sur base de cette analyse, nous montrons que le mécanisme en place est en fait proche d'une prime de rachat garantie (FiP). Dans un contexte où le prix de gros est à la hausse, nous pensons que ce mécanisme devrait rapidement évoluer, au risque sinon pour la collectivité de devoir assumer une facture importante pour pouvoir continuer à soutenir les énergies renouvelables.

\section{MÉCANISME DE SOUTIEN AUX ÉNERGIES RENOUVELABLES}

La stratégie Europe 2020 vise à accroître la part des énergies renouvelables dans le mix énergétique global. Pour favoriser la production d'électricité issue de sources d'énergie renouvelable, les États membres de l'Union européenne ont mis en place différents dispositifs de soutien. Le mécanisme peut soit fixer 
un objectif en terme de quantité d'énergie renouvelable à produire et laisser le marché déterminer le prix à payer pour atteindre cet objectif. Les enchères de quotas d'émission ou de certificats verts en sont des exemples où le prix se détermine grâce à un mécanisme concurrentiel. Alternativement, le mécanisme peut déterminer le prix du soutien et laisser les quantités produites s'ajuster par la loi de l'offre. Les tarifs de rachat garantis (feed-in tariff ou FiT) et les primes de rachat garanties (feed-in-premium ou FiP) se fondent sur des prix fixes et régulés. Les deux types de mécanismes ne sont pas équivalents notamment dans un environnement incertain : voir Menanteau et al. (2003) pour une comparaison théorique et Ringel (2006) pour des comparaisons entre pays.

\subsection{Tarifs et primes de rachat garantis (FiT et FiP)}

Le tarif de rachat garanti oblige les fournisseurs d'énergie à acheter l'énergie renouvelable produite à un prix garanti pour une période donnée. Le producteur a donc la certitude de pouvoir vendre son électricité à un prix défini à l'avance. II est donc assuré contre les fluctuations du prix de l'énergie sur le marché et contre l'évolution technologique qui pourrait rendre son unité de production obsolète. La prime de rachat garantie fonctionne sur le même principe, à la seule différence que l'énergie est vendue sur le marché augmenté d'une prime dont le montant est fixé à l'avance. La FiP n'assure donc plus le producteur contre les fluctuations du marché de l'énergie. Elle lui garantit néanmoins un avantage par rapport aux technologies " classiques".

Les tarifs et primes de rachat garantis doivent être définis séparément pour chaque technologie, le coût au kWh n'étant pas nécessairement le même pour l'éolien et le solaire par exemple. De ce fait, les différentes technologies ne sont pas en concurrence entre elles. Par ailleurs, les tarifs peuvent être revus périodiquement pour s'adapter aux évolutions technologiques et économiques. Le mécanisme nécessite des modifications régulières pour lesquelles le régulateur doit collecter de nombreuses informations pour calibrer au mieux le prix régulé aux conditions de marché.

\subsection{Certificats verts (CV)}

Avec le mécanisme de certificats verts, l'énergie produite est vendue au prix du marché. Les producteurs reçoivent pour soutenir leur production des certificats verts au prorata de l'électricité produite, avec un taux d'octroi qui peut être différent d'une technologie à l'autre. Ces certificats sont nécessaires aux fournisseurs pour remplir leurs obligations de quota et les certificats peuvent s'échanger sur le marché. C'est le prix du certificat sur le marché qui détermine le montant financier du soutien accordé à la production. Le prix du certificat n'étant pas différencié par filière, les différentes technologies sont donc en concurrence entre elles. Avec les certificats verts, les producteurs ne sont pas assurés contre le risque technologique. Ce mécanisme ne nécessite pas a priori autant d'information que 
les mécanismes basés sur le prix puisque le subside octroyé se détermine par un mécanisme de marché. Le risque pour les producteurs est cependant plus important. Ce risque peut-être limité en fixant des quotas de CV a priori. Dans cette hypothèse, on peut utiliser comme alternative aux CV, la mise aux enchères de la production d'énergie renouvelable. Dans ce cas, le niveau de soutien est également déterminé par un mécanisme de marché.

\section{LE MARCHÉ DES CV EN WALLONIE}

\subsection{Organisation du marché}

La Wallonie a mis en place en 2003 un mécanisme de certificats verts pour soutenir la production d'électricité issue de sources énergétiques renouvelables. Le marché des certificats verts s'organise de la manière suivante. D'un côté, les producteurs d'énergie issue de sources renouvelables reçoivent des certificats verts en fonction de leur production. Les CV sont, en principe, octroyés pour une durée de 10 ans. Des CV sont octroyés pour chaque MWh renouvelable produit. Le nombre de certificats varie d'une filière à l'autre et il est calculé sur base des économies de $\mathrm{CO}_{2}$ réalisées. Nous reviendrons sur les révisions du taux d'octroi plus loin dans cette section. Le tableau 1, issu du rapport 2016 de la CWaPE sur l'évolution du marché des certificats verts (p. 55), présente le taux d'octroi effectif par filière pour l'année 2016.

\section{Tableau 1. Taux d'octroi de CV par filière}

\begin{tabular}{|l|c|}
\hline Filière & CV/MWh \\
\hline Éolien & 1 \\
\hline Biomasse & 1,48 \\
\hline Solaire & 5,7 \\
\hline Hydraulique & 0,39 \\
\hline Cogénération fossile & 0,1 \\
\hline
\end{tabular}

Du côté de la demande, les fournisseurs d'énergie sont soumis à une obligation de quota. Ils doivent certifier qu'un certain pourcentage de l'électricité délivrée est issu de sources renouvelables. Ce pourcentage était de $3 \%$ en 2003 et il a augmenté considérablement pour atteindre $34 \%$ en 2017. Certaines exceptions sont prévues et le quota effectif est inférieur au quota nominal mentionné. Les CV sont utilisés par les fournisseurs d'électricité pour certifier la provenance de l'énergie.

Pour permettre aux producteurs d'atteindre leurs obligations de quota, les CV sont échangeables sur un marché spécialement créé pour cela avec la particularité de posséder un prix plancher $(65 €)$ et un prix plafond (100 €). Le prix plafond correspond à l'amende administrative pour certificats manquants. Le prix 
plancher de $65 €$ correspond à l'obligation de rachat imposée au GRT (Elia). Dans les faits, le prix des CV tend à rester dans cet intervalle, ce qui limite le risque à charge du producteur. Les CV rachetés par Elia sont répercutés sur la facture du consommateur via une redevance spécifique. Le mécanisme qui fut mis en place en 2003 a été modifié deux fois, en 2007 avec le plan SOLWATT et en 2014 avec une nouvelle procédure d'octroi des CV et un nouveau calcul des taux d'octroi.

Le taux d'octroi pour la filière photovoltaïque a subi plusieurs modifications. Avant 2007, il était lié aux économies de $\mathrm{CO}_{2}$ et fixé comme pour l'éolien à $1 \mathrm{CV}$ par MWh, un taux auquel la technologie n'était pas compétitive. En 2007, le plan SOLWATT destiné à promouvoir le solaire photovoltaïque de petite puissance $(<10 \mathrm{kWC}$ ) a modifié le taux d'octroi de 1 à 7 CV par MWh et a prolongé la durée d'octroi à 15 ans. À partir de 2011, la durée puis le taux d'octroi ont tous les deux été modifiés pour s'adapter aux conditions du marché ${ }^{3}$.

En 2014, le gouvernement wallon a mis fin au plan SOLWATT si bien que les nouvelles installations photovoltaïques de petite puissance installées après cette date ne sont plus soutenues par le mécanisme de CV ${ }^{4}$. Les installations photovoltaïques d'une puissance supérieure à $10 \mathrm{kWc}$ et les installations installées avant 2014 continuent de bénéficier de certificats verts. La réforme de 2014 a changé les conditions et les taux d'octroi de CV. Pour éviter un emballement du système, un quota de CV annuel est disponible pour chaque filière et les nouveaux producteurs doivent réserver les $\mathrm{CV}$. De cette manière, on évite un développement trop important d'une filière et l'offre de CV est maintenue sous contrôle. Les taux d'octroi ont également été révisés de manière assez fondamentale. Alors qu'auparavant le taux dépendait exclusivement des économies de $\mathrm{CO}_{2}$ (sauf pour le programme SOLWATT), il dépend maintenant de facteurs économiques comme le prix de marché de l'énergie ou bien le coût de la technologie. Un coefficient économique est calculé sur base de ces paramètres et entre en ligne de compte pour le calcul du taux d'octroi. Ce que l'on doit finalement retenir, c'est que les paramètres déterminant le taux d'octroi s'ajustent de manière à garantir un taux de rentabilité défini à l'installation.

\section{2 Évolution du marché 5}

Sur la période 2003-2017, plus 58,64 millions de CV ont été octroyés, 23 millions pour les installations solaires dans le cadre du plan SOLWATT et 35 millions pour les autres filières. Sur cette même période, le quota de CV effectif était de 37 millions de CV. II y a donc un surplus de près de 21 millions de CV. Si ceux-ci

3. Pour l'année 2016, le taux d'octroi effectif pour les installations soutenues dans le cadre du programme SOLWATT était de 6,5 CV/MWh.

4. Le gouvernement wallon a remplacé le plan SOLWATT par un autre mécanisme de soutien appelé QUALIWATT. Les installations reçoivent une prime en fonction de leur puissance de manière à garantir son rendement. Le plan QUALIWATT s'est terminé en 2018 et le seul soutien octroyé est le mécanisme de compensation (le compteur qui tourne à l'envers).

5. Les données utilisées dans cette section proviennent du rapport annuel spécifique relatif au marché des certificats verts publié annuellement par la CWaPE, le régulateur wallon de l'énergie qui organise et supervise le marché. 
sont valorisés au prix plancher de $65 €$, cela représente un coût de 1,36 milliard d'euros. L'excès d'offre sur le marché correspond à peu de choses près aux CV accordés dans le cadre du plan SOLWATT, même s'il faut noter que, depuis 2014, les CV de la filière solaire sont nécessaires pour atteindre les obligations de quota, ce qui n'était pas le cas précédemment.

Figure 1. Offre et demande de CV, 2003-2017

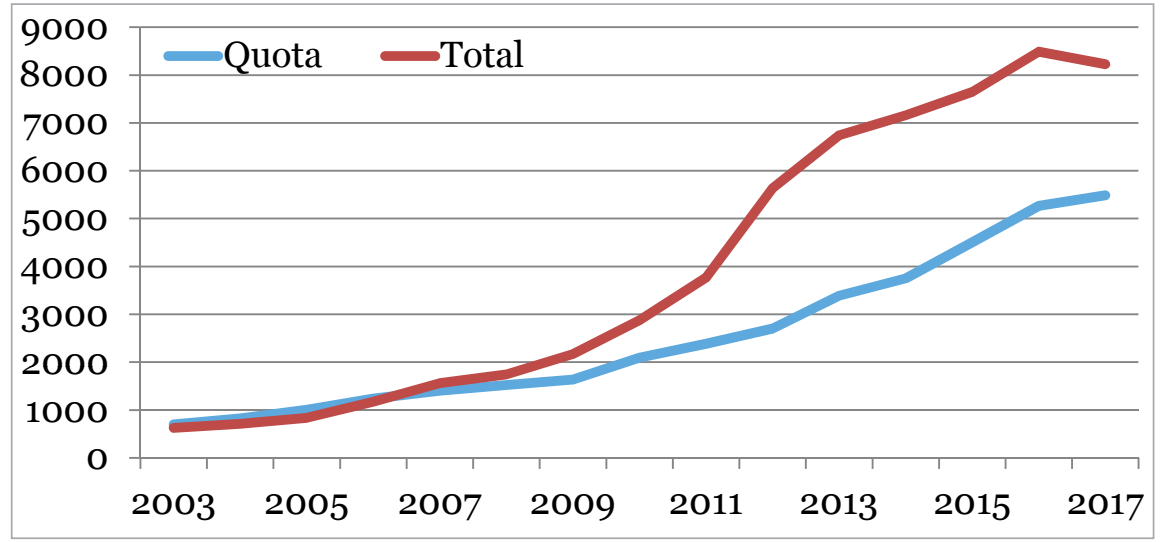

Figure 2. Prix du CV, 2003-2017

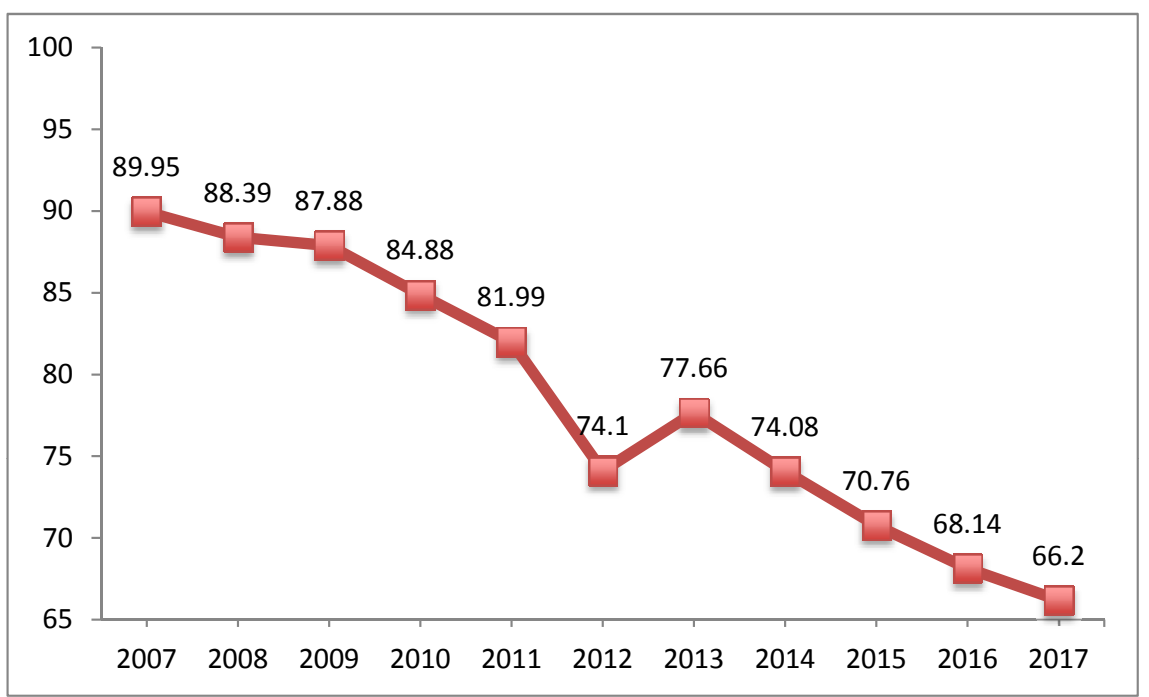


Figure 3. Octroi de CV en pourcentage du quota annuel, 2003-2017

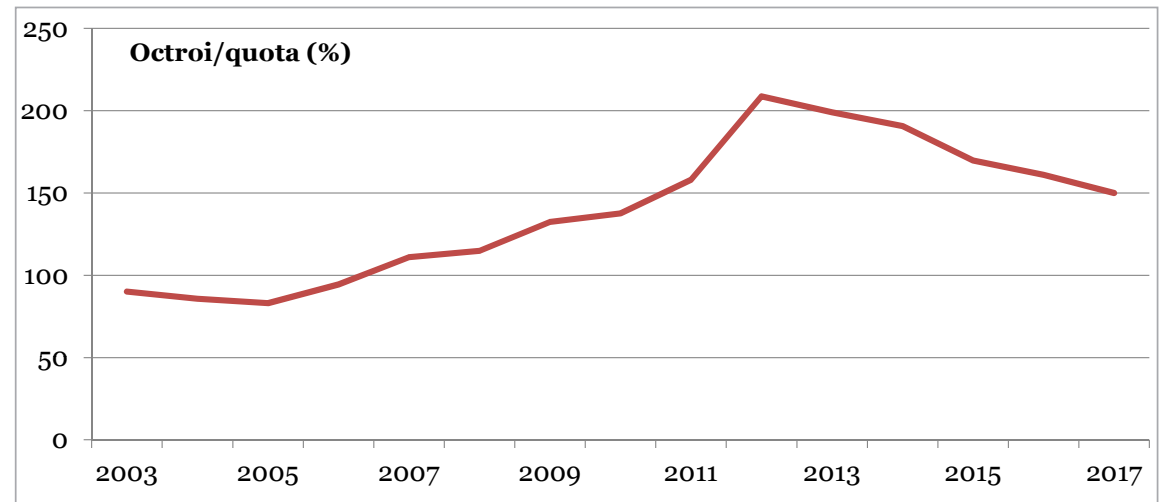

Les deux graphiques suivants reprennent l'évolution de l'offre et de la demande sur le marché (figure 1) et l'évolution du prix du CV (figure 2) depuis la mise en place du mécanisme en 2003. Les graphiques montrent de façon très claire que le marché est, à partir de 2007, en déséquilibre structurel avec une offre supérieure à la demande. La figure 3 illustre ce déséquilibre en exprimant le nombre de CV octroyés en pourcentage du quota. En termes relatifs, le déséquilibre culmine en 2012 avec plus du double de CV octroyés par rapport au quota. Depuis 2013, le déséquilibre s'est réduit mais il reste conséquent et les prévisions du régulateur montrent que le déséquilibre structurel n'est pas prêt de se résorber ${ }^{6}$. Avec un tel excès d'offre sur le marché, il n'est pas étonnant que le prix du CV n'ait fait que chuter et il est aujourd'hui proche du prix plancher de $65 €$.

\section{CONCLUSIONS}

Le mécanisme de certificats verts wallons ne fonctionne plus correctement. D'une part, l'excès de CV sur le marché a créé une dette importante qu'il y a lieu d'épurer, soit en la répercutant intégralement sur les consommateurs, avec éventuellement un lissage dans le temps, soit en la partageant avec les producteurs, ce qui peut se faire par exemple en diminuant le prix de rachat garanti pour certaines filières. D'autre part, le prix du CV qui est, en principe, un prix de marché qui doit guider les décisions d'investissement en signalant la valeur de marché de la prime au renouvelable ne joue plus son rôle de signal. Dans les faits, le prix est durablement fixé à son prix plancher fixé administrativement et il n'a dès lors plus aucune signification. La conséquence est que le soutien reçu par chaque filière de production s'apparente à une prime garantie dont le montant par MWh est

6. Dans son avis du 4 décembre 2018 (https://www.cwape.be/docs/?doc=3948), le régulateur prévoit, à politique inchangée, un marché en déséquilibre jusqu'en 2030 avec une offre systématiquement supérieure à la demande. 
donné par le taux d'octroi (cf. tableau 1) multiplié par le prix plancher du CV, soit $65 €$. Le mécanisme ne met plus en concurrence les technologies et l'incertitude relative au niveau de soutien effectif a disparu. Le système wallon a évolué d'un mécanisme basé sur les quantités à un mécanisme basé sur les prix, de certificats verts échangeables à une prime de rachat garantie.

Celle-ci est actuellement fixée à $65 € / M W h$ pour l'éolien. À titre de comparaison, le tarif d'achat en France est de $82 € / \mathrm{MWh}$ et en Allemagne de $73 € / \mathrm{MWh}$. Sachant que le prix de gros de l'électricité est aux alentours de $35 € / M W h$, le soutien accordé reste particulièrement généreux et s'apparente à une redistribution de rente. Dans une perspective d'équité, il nous semble donc important d'ajuster régulièrement le niveau de soutien à la réalité du marché. C'est le rôle du coefficient économique dans la formule de calcul du taux d'octroi qui réduit celui-ci, périodiquement, en fonction de paramètres capturant l'évolution de la technologie et du marché.

Par ailleurs, comme le mécanisme actuel (réformé en 2014) est caractérisé par un quota de certificats par filière, il n'y a plus aucun mécanisme de marché dans le soutien aux énergies renouvelables en Wallonie. La quantité à produire et le niveau de soutien par filière sont fixés en dehors du marché de manière administrative, peu propice à encourager l'innovation et à donner des signaux corrects aux marchés. Le maintien de mécanismes de marché nous semble primordial pour encourager le soutien au renouvelable tout en limitant le plus possible les subsides octroyés. La détermination du niveau de soutien par une autorité administrative nécessite de nombreuses informations et une grande flexibilité. Dans ce contexte, l'utilisation de procédure concurrentielle nous semble plus appropriée.

La proposition du régulateur du marché (CWaPE, 2018) prévoit de mettre fin progressivement au système de CV et de subventionner les nouvelles installations au moyen d'une prime de rachat (FiP). La prime serait déterminée administrativement pour garantir le rendement de l'installation, en faisant exception des procédures concurrentielles pour des installations de grande taille dans certaines filières (photovoltaïque de grande puissance $>1 \mathrm{MW}$ et biomasse solide $>2 \mathrm{MW}$ ). Le gouvernement wallon a quant à lui proposé un projet d'arrêté et un projet de décret qui prévoient le maintien du mécanisme de certificats verts tout en modifiant à la hausse le quota et à la baisse le taux d'octroi de manière à faire converger, à terme, l'offre et la demande. ${ }^{7}$ Les propositions du gouvernement incluent également un système de portage pour les CV émis en excès et dont le coût n'a pas encore été répercuté sur les consommateurs. Ce mécanisme permet de lisser le coût de la surcharge facturée aux consommateurs sur plusieurs années. L'absence de mécanisme de marché dans la proposition de réforme du gouvernement et le maintien d'un système où le soutien est déterminé exclusivement de manière administrative nous semblent problématiques.

7. À ce jour, le projet de décret a été approuvé en $3^{e}$ lecture par le gouvernement et doit être voté au Parlement de Wallonie. Le projet d'arrêté gouvernemental doit être approuvé en $3^{e}$ lecture par le gouvernement wallon. Ces deux projets ont été adoptés en avril 2019. 


\section{RÉFÉRENCES}

Boccard, N., Gautier, A. (2015), "Le coût de l'énergie verte en Wallonie 2003-2012 », Reflets et perspectives de la vie économique, 54 (1-2), 71-85.

CWAPE (2003-2017) Rapports annuels spécifiques sur l'évolution du marché des certificats verts.

CWAPE (2018) Proposition de révision du mécanisme de soutien à la production d'énergie verte en Wallonie (https://www.cwape.be/docs/?doc=3592).

Menanteau, P., Finon, D., Lamy, M. (2003), "Prices versus quantities: Choosing policies for promoting the development of renewable energy ", Energy Policy, 31, 799-812.

RINGEL, M. (2006), "Fostering the use of renewable energies in the European Union: The race between feed-in tariffs and green certificates ", Renewable Energy, 31(1), 1-17. 